\title{
Existence Results for Singular Boundary Value Problem of Nonlinear Fractional Differential Equation
}

\author{
Yujun Cui \\ Department of Mathematics, Shandong University of Science and Technology, Qingdao 266510, China \\ Correspondence should be addressed to Yujun Cui, cyj720201@163.com
}

Received 27 December 2010; Revised 2 March 2011; Accepted 3 March 2011

Academic Editor: Elena Braverman

Copyright (C) 2011 Yujun Cui. This is an open access article distributed under the Creative Commons Attribution License, which permits unrestricted use, distribution, and reproduction in any medium, provided the original work is properly cited.

By applying a fixed point theorem for mappings that are decreasing with respect to a cone, this paper investigates the existence of positive solutions for the nonlinear fractional boundary value problem: $D_{0^{+}}^{\alpha} u(t)+f(t, u(t))=0,0<t<1, u(0)=u^{\prime}(0)=u^{\prime}(1)=0$, where $2<\alpha<3, D_{0^{+}}^{\alpha}$ is the Riemann-Liouville fractional derivative.

\section{Introduction}

Many papers and books on fractional calculus differential equation have appeared recently. Most of them are devoted to the solvability of the linear initial fractional equation in terms of a special function [1-4]. Recently, there has been significant development in the existence of solutions and positive solutions to boundary value problems for fractional differential equations by the use of techniques of nonlinear analysis (fixed point theorems, LeraySchauder theory, etc.), see $[5,6]$ and the references therein.

In this paper, we consider the following boundary value problems of the nonlinear fractional differential equation

$$
\begin{gathered}
D_{0^{+}}^{\alpha} u(t)+f(t, u(t))=0, \quad 0<t<1,2<\alpha<3, \\
u(0)=u^{\prime}(0)=u^{\prime}(1)=0
\end{gathered}
$$

where $D_{0^{+}}^{\alpha}$ is the standard Riemann-Liouville fractional derivative and $f(t, x)$ is singular at 
$x=0$. Our assumptions throughout are

$\left(\mathrm{H}_{1}\right) f(t, x):(0,1) \times(0, \infty) \rightarrow[0, \infty)$ is continuous,

$\left(\mathrm{H}_{2}\right) f(t, x)$ is decreasing in $x$, for each fixed $t$,

$\left(\mathrm{H}_{3}\right) \lim _{x \rightarrow 0^{+}} f(t, x)=\infty$ and $\lim _{x \rightarrow \infty} f(t, x)=0$, uniformly on compact subsets of $(0,1)$, and

$\left(\mathrm{H}_{4}\right) 0<\int_{0}^{1} f\left(t, q_{\theta}(t)\right) d t<\infty$ for all $\theta>0$ and $q_{\theta}$ as defined in (3.1).

The seminal paper by Gatica et al. [7] in 1989 has had a profound impact on the study of singular boundary value problems for ordinary differential equations (ODEs). They studied singularities of the type in $\left(\mathrm{H}_{1}\right)-\left(\mathrm{H}_{4}\right)$ for second order Sturm-Louiville problems, and their key result hinged on an application of a particular fixed point theorem for operators which are decreasing with respect to a cone. Various authors have used these techniques to study singular problems of various types. For example, Henderson and Yin [8] as well as Eloe and Henderson $[9,10]$ have studied right focal, focal, conjugate, and multipoint singular boundary value problems for ODEs. However, as far as we know, no paper is concerned with boundary value problem for fractional differential equation by using this theorem. As a result, the goal of this paper is to fill the gap in this area.

Motivated by the above-mentioned papers and [11], the purpose of this paper is to establish the existence of solutions for the boundary value problem (1.1) by the use of a fixed point theorem used in [7, 11]. The paper has been organized as follows. In Section 2, we give basic definitions and provide some properties of the corresponding Green's function which are needed later. We also state the fixed point theorem from [7] for mappings that are decreasing with respect to a cone. In Section 3, we formulate two lemmas which establish a priori upper and lower bounds on solutions of (1.1). We then state and prove our main existence theorem.

For fractional differential equation and applications, we refer the reader to [1-3]. Concerning boundary value problems (1.1) with ordinary derivative (not fractional one), we refer the reader to $[12,13]$.

\section{Some Preliminaries and a Fixed Point Theorem}

For the convenience of the reader, we present here the necessary definitions from fractional calculus theory. These definitions and properties can be found in the literature.

Definition 2.1 (see [3]). The Riemann-Liouville fractional integral of order $\alpha>0$ of a function $f:(0, \infty) \rightarrow R$ is given by

$$
I_{0+}^{\alpha} f(t)=\frac{1}{\Gamma(\alpha)} \int_{0}^{t}(t-s)^{\alpha-1} f(s) d s
$$

provided that the right-hand side is pointwise defined on $(0, \infty)$.

Definition 2.2 (see [3]). The Riemann-Liouville fractional derivative of order $\alpha>0$ of a continuous function $f:(0, \infty) \rightarrow R$ is given by

$$
D_{0^{+}}^{\alpha} f(t)=\frac{1}{\Gamma(n-\alpha)}\left(\frac{d}{d t}\right)^{n} \int_{0}^{t} \frac{f(s)}{(t-s)^{\alpha-n+1}} d s,
$$


where $n-1 \leq \alpha<n$, provided that the right-hand side is pointwise defined on $(0, \infty)$.

Definition 2.3. By a solution of the boundary value problem (1.1) we understand a function $u \in C[0,1]$ such that $D_{0^{+}}^{\alpha} u$ is continuous on $(0,1)$ and $u$ satisfies (1.1).

Lemma 2.4 (see [3]). Assume that $u \in C(0,1) \cap L(0,1)$ with a fractional derivative of order $\alpha>0$ that belongs to $C(0,1) \cap L(0,1)$. Then

$$
I_{0+}^{\alpha} D_{0+}^{\alpha} u(t)=u(t)+c_{1} t^{\alpha-1}+c_{2} t^{\alpha-2}+\cdots+c_{N} t^{\alpha-N}
$$

for some $c_{i} \in R, i=1, \ldots, N, N=[\alpha]$.

Lemma 2.5. Given $f \in C[0,1]$, and $2<\alpha<3$, the unique solution of

$$
\begin{gathered}
D_{0^{+}}^{\alpha} u(t)+f(t)=0, \quad 0<t<1, \\
u(0)=u^{\prime}(0)=u^{\prime}(1)=0
\end{gathered}
$$

is

$$
u(t)=\int_{0}^{1} G(t, s) f(s) d s,
$$

where

$$
G(t, s)= \begin{cases}\frac{t^{\alpha-1}(1-s)^{\alpha-2}-(t-s)^{\alpha-1}}{\Gamma(\alpha)}, & 0 \leq s \leq t \leq 1 \\ \frac{t^{\alpha-1}(1-s)^{\alpha-2}}{\Gamma(\alpha)}, & 0 \leq t \leq s \leq 1\end{cases}
$$

Proof. We may apply Lemma 2.4 to reduce (2.4) to an equivalent integral equation

$$
u(t)=-I_{0+}^{\alpha} f(t)+c_{1} t^{\alpha-1}+c_{2} t^{\alpha-2}+c_{3} t^{\alpha-3}
$$

for some $c_{i} \in R, i=1,2,3$. From $u(0)=u^{\prime}(0)=u^{\prime}(1)=0$, one has

$$
c_{1}=\int_{0}^{1} \frac{(1-s)^{\alpha-2}}{\Gamma(\alpha)} f(s) d s, \quad c_{2}=c_{3}=0 .
$$

Therefore, the unique solution of problem (2.4) is

$$
\begin{aligned}
u(t) & =\int_{0}^{1} \frac{t^{\alpha-1}(1-s)^{\alpha-2}}{\Gamma(\alpha)} f(s) d s-\frac{1}{\Gamma(\alpha)} \int_{0}^{t}(t-s)^{\alpha-1} f(s) d s \\
& =\int_{0}^{t}\left[\frac{t^{\alpha-1}(1-s)^{\alpha-2}-(t-s)^{\alpha-1}}{\Gamma(\alpha)}\right] f(s) d s+\int_{t}^{1} \frac{t^{\alpha-1}(1-s)^{\alpha-2}}{\Gamma(\alpha)} f(s) d s \\
& =\int_{0}^{1} G(t, s) f(s) d s .
\end{aligned}
$$


Lemma 2.6. The function $G(t, s)$ defined by (2.6) satisfies the following conditions:

(i) $G(t, s)>0,0<t, s<1$,

(ii) $q(t) G(1, s) \leq G(t, s) \leq G(1, s)=s(1-s)^{\alpha-2} /(\Gamma(\alpha))$ for $0 \leq t, s \leq 1$, where $q(t)=t^{\alpha-1}$.

Proof. Observing the expression of $G(t, s)$, it is clear that $G(t, s)>0$ for $0<t, s<1$. For given $s \in(0,1), G(t, s)$ is increasing with respect to $t$. Consequently, $G(t, s) \leq G(1, s)$ for $0 \leq t, s \leq 1$. If $s \leq t$, we have

$$
\begin{aligned}
G(t, s) & =\frac{t(t-t s)^{\alpha-2}-(t-s)(t-s)^{\alpha-2}}{\Gamma(\alpha)} \\
& \geq \frac{t(t-t s)^{\alpha-2}-(t-s)(t-t s)^{\alpha-2}}{\Gamma(\alpha)} \\
& =\frac{s t^{\alpha-2}(1-s)^{\alpha-2}}{\Gamma(\alpha)} \geq \frac{s t^{\alpha-1}(1-s)^{\alpha-2}}{\Gamma(\alpha)}=q(t) G(1, s) .
\end{aligned}
$$

If $t \leq s$, we have

$$
G(t, s)=\frac{t^{\alpha-1}(1-s)^{\alpha-2}}{\Gamma(\alpha)} \geq \frac{s t^{\alpha-1}(1-s)^{\alpha-2}}{\Gamma(\alpha)}=q(t) G(1, s) .
$$

Let $E$ be a Banach space, $P \subset E$ be a cone in $E$. Every cone $P$ in $E$ defines a partial ordering in $E$ given by $x \leq y$ if and only if $y-x \in P$. If $x \leq y$ and $x \neq y$, we write $x<y$. A cone $P$ is said to be normal if there exists a constant $N>0$ such that $\theta \leq x \leq y$ implies $\|x\| \leq N\|y\|$. If $P$ is normal, then every order interval $[x, y]=\{z \in E \mid x \leq z \leq y\}$ is bounded. For the concepts and properties about the cone theory we refer to $[14,15]$.

Next we state the fixed point theorem due to Gatica et al. [7] which is instrumental in proving our existence results.

Theorem 2.7 (Gatica-Oliker-Waltman fixed point theorem). Let $E$ be a Banach space, $P \subset E$ be a normal cone, and $D \subset P$ be such that if $x, y \in D$ with $x \leq y$, then $[x, y] \subset D$. Let $T: D \rightarrow P$ be a continuous, decreasing mapping which is compact on any closed order interval contained in $D$, and suppose there exists an $x_{0} \subset D$ such that $T^{2} x_{0}$ is defined (where $T^{2} x_{0}=T\left(T x_{0}\right)$ ) and $T x_{0}, T^{2} x_{0}$ are order comparable to $x_{0}$. Then $T$ has a fixed point in $D$ provided that either:

(i) $T x_{0} \leq x_{0}$ and $T^{2} x_{0} \leq x_{0}$;

(ii) $x_{0} \leq T x_{0}$ and $x_{0} \leq T^{2} x_{0}$; or

(iii) The complete sequence of iterates $\left\{T^{n} x_{0}\right\}_{n=0}^{\infty}$ is defined and there exists $y_{0} \in D$ such that $T y_{0} \in D$ with $y_{0} \leq T^{n} x_{0}$ for all $n \in \mathrm{N}$.

\section{Main Results}

In this section, we apply Theorem 2.7 to a sequence of operators that are decreasing with respect to a cone. These obtained fixed points provide a sequence of iterates which converges to a solution of (1.1). 
Let the Banach space $E=C[0,1]$ with the maximum norm $\|u\|=\max _{t \in[0,1]}|u(t)|$, and let $P=\{u \in E \mid u(t) \geq 0, t \in[0,1]\}$. $P$ is a norm cone in $E$. For $\theta>0$, let

$$
q_{\theta}(t)=\theta \cdot q(t)
$$

where $q(t)$ is given in Lemma 2.6. Define $D \subset P$ by

$$
D=\left\{u \in P \mid \exists \theta(u)>0 \text { such that } u(t) \geq q_{\theta}(t), t \in[0,1]\right\},
$$

and the integral operator $T: D \rightarrow P$ by

$$
(T u)(t)=\int_{0}^{1} G(t, s) f(s, u(s)) d s,
$$

where $G(t, s)$ is given in (2.6). It suffices to define $D$ as above, since the singularity in $f$ precludes us from defining $T$ on all of $P$. Furthermore, it can easily be verified that $T$ is well defined. In fact, note that for $u \in D$ there exists $\theta(u)>0$ such that $u(t) \geq q_{\theta}(t)$ for all $t \in[0,1]$. Since $f(t, x)$ decreases with respect to $x$, we see $f(t, u(t)) \leq f\left(t, q_{\theta}(t)\right)$ for $t \in[0,1]$. Thus,

$$
0 \leq \int_{0}^{1} G(t, s) f(s, u(s)) d s \leq \int_{0}^{1} f\left(s, q_{\theta}(s)\right) d s<\infty
$$

Similarly, $T$ is decreasing with respect to $D$.

Lemma 3.1. $u \in D$ is a solution of (1.1) if and only if $T u=u$.

Proof. One direction of the lemma is obviously true. To see the other direction, let $u \in D$. Then $(T u)(t)=\int_{0}^{1} G(t, s) f(s, u(s)) d s$, and Tu satisfies (1.1). Moreover, by Lemma 2.6, we have

$$
\begin{aligned}
(T u)(t) & =\int_{0}^{1} G(t, s) f(s, u(s)) d s \\
& \geq q(t) \int_{0}^{1} G(1, s) f(s, u(s)) d s=q(t)\|T u\|, \quad \forall t \in[0,1] .
\end{aligned}
$$

Thus, there exists some $\theta(T u)$ such that $(T u)(t) \geq q_{\theta}(t)$, which implies that $T u \in D$. That is, $T: D \rightarrow D$.

We now present two lemmas that are required in order to apply Theorem 2.7. The first establishes a priori upper bound on solutions, while the second establishes a priori lower bound on solutions.

Lemma 3.2. If $f$ satisfies $\left(H_{1}\right)-\left(H_{4}\right)$, then there exists an $S>0$ such that $\|u\| \leq S$ for any solution $u \in D$ of (1.1). 
Proof. For the sake of contradiction, suppose that the conclusion is false. Then there exists a sequence $\left\{u_{n}\right\}_{n=1}^{\infty}$ of solutions to (1.1) such that $\left\|u_{n}\right\| \leq\left\|u_{n+1}\right\|$ with $\lim _{n \rightarrow \infty}\left\|u_{n}\right\|=\infty$. Note that for any solution $u_{n} \in D$ of (1.1), by (3.5), we have

$$
u_{n}(t)=\left(T u_{n}\right)(t) \geq q(t)\left\|u_{n}\right\|, \quad t \in[0,1], n \geq 1
$$

Then, assumptions $\left(\mathrm{H}_{2}\right)$ and $\left(\mathrm{H}_{4}\right)$ yield, for $0 \leq t \leq 1$ and all $n \geq 1$,

$$
\begin{aligned}
u_{n}(t) & =\left(T u_{n}\right)(t)=\int_{0}^{1} G(t, s) f\left(s, u_{n}(s)\right) d s \\
& \leq \frac{1}{\Gamma(\alpha)} \int_{0}^{1} s(1-s)^{\alpha-2} f\left(s, q_{\left\|u_{1}\right\|}(s)\right) d s=N
\end{aligned}
$$

for some $0<N<+\infty$. In particular, $\left\|u_{n}\right\| \leq N$, for all $n \geq 1$, which contradicts $\lim _{n \rightarrow \infty}\left\|u_{n}\right\|=$ $\infty$.

Lemma 3.3. If $f$ satisfies $\left(H_{1}\right)-\left(H_{4}\right)$, then there exists an $R>0$ such that $\|u\| \geq R$ for any solution $u \in D$ of (1.1).

Proof. For the sake of contradiction, suppose $u_{n}(t) \rightarrow 0$ uniformly on $[0,1]$ as $n \rightarrow \infty$. Let $M=\inf \{G(t, s):(t, s) \in[1 / 4,3 / 4] \times[1 / 4,3 / 4]\}>0$. From $\left(\mathrm{H}_{3}\right)$, we see that $\lim _{x \rightarrow 0^{+}} f(t, x)=$ $\infty$ uniformly on compact subsets of $(0,1)$. Hence, there exists some $\delta>0$ such that for $t \in$ $[1 / 4,3 / 4]$ and $0<x<\delta$, we have $f(t, x) \geq 2 / M$. On the other hand, there exists an $n_{0} \in N$ such that $n \geq n_{0}$ implies $0<u_{n}(t)<\delta / 2$, for $t \in(0,1)$. So, for $t \in[1 / 4,3 / 4]$ and $n \geq n_{0}$,

$$
\begin{aligned}
u_{n}(t) & =\left(T u_{n}\right)(t)=\int_{0}^{1} G(t, s) f\left(s, u_{n}(s)\right) d s \geq \int_{1 / 4}^{3 / 4} G(t, s) f\left(s, u_{n}(s)\right) d s \\
& \geq M \int_{1 / 4}^{3 / 4} f\left(s, \frac{\delta}{2}\right) d s \geq M \int_{1 / 4}^{3 / 4} \frac{2}{M} d s=1 .
\end{aligned}
$$

But this contradicts the assumption that $\left\|u_{n}\right\| \rightarrow 0$ uniformly on $[0,1]$ as $n \rightarrow \infty$. Hence, there exists an $R>0$ such that $R \leq\|u\|$.

We now present the main result of the paper.

Theorem 3.4. If $f$ satisfies $\left(H_{1}\right)-\left(H_{4}\right)$, then (1.1) has at least one positive solution.

Proof. For each $n \geq 1$, defined $v_{n}:[0,1] \rightarrow[0,+\infty)$ by

$$
v_{n}(t)=\int_{0}^{1} G(t, s) f(s, n) d s
$$


By conditions $\left(\mathrm{H}_{1}\right)-\left(\mathrm{H}_{4}\right)$, for $n \geq 1$,

$$
\begin{gathered}
0<v_{n+1}(t) \leq v_{n}(t), \quad \text { on }(0,1], \\
\lim _{n \rightarrow \infty} v_{n}(t)=0 \quad \text { uniformly on }[0,1] .
\end{gathered}
$$

Now define a sequence of functions $f_{n}:(0,1) \times[0,+\infty), n \geq 1$, by

$$
f_{n}(t, x)=f\left(t, \max \left\{x, v_{n}(t)\right\}\right) .
$$

Then, for each $n \geq 1, f_{n}$ is continuous and satisfies $\left(\mathrm{H}_{2}\right)$. Furthermore, for $n \geq 1$,

$$
\begin{gathered}
f_{n}(t, x) \leq f(t, x) \quad \text { on }(0,1) \times(0,+\infty), \\
f_{n}(t, x) \leq f\left(t, v_{n}(t)\right) \quad \text { on }(0,1) \times(0,+\infty) .
\end{gathered}
$$

Note that $f_{n}$ has effectively "removed the singularity" in $f(t, x)$ at $x=0$, then we define a sequence of operators $T_{n}: P \rightarrow P, n \geq 1$, by

$$
\left(T_{n} u\right)(t)=\int_{0}^{1} G(t, s) f_{n}(s, u(s)) d s, u \in P .
$$

From standard arguments involving the Arzela-Ascoli Theorem, we know that each $T_{n}$ is in fact a compact mapping on $P$. Furthermore, $T_{n}(0) \geq 0$ and $T_{n}^{2}(0) \geq 0$. By Theorem 2.7, for each $n \geq 1$, there exists $u_{n} \in P$ such that $T_{n} u_{n}(x)=u_{n}(t)$ for $t \in[0,1]$. Hence, for each $n \geq 1, u_{n}$ satisfies the boundary conditions of the problem. In addition, for each $u_{n}$,

$$
\begin{aligned}
\left(T_{n} u_{n}\right)(t) & =\int_{0}^{1} G(t, s) f_{n}\left(s, u_{n}(s)\right) d s=\int_{0}^{1} G(t, s) f_{n}\left(s, \max \left\{u_{n}(s), v_{n}(s)\right\}\right) d s \\
& \leq \int_{0}^{1} G(t, s) f_{n}\left(s, v_{n}(s)\right) d s \leq T v_{n}(t)
\end{aligned}
$$

which implies

$$
u_{n}(t)=\left(T_{n} u_{n}\right)(t) \leq T v_{n}(t), \quad t \in[0,1], n \in \mathrm{N}
$$

Arguing as in Lemma 3.2 and using (3.11), it is fairly straightforward to show that there exists an $S>0$ such that $\left\|u_{n}\right\| \leq S$ for all $n \in \mathrm{N}$. Similarly, we can follow the argument of Lemma 3.3 and (3.5) to show that there exists an $R>0$ such that

$$
u_{n}(t) \geq q(t) R, \quad \text { on }[0,1], \text { for } n \geq 1
$$

Since $T: D \rightarrow D$ is a compact mapping, there is a subsequence of $\left\{T u_{n}\right\}$ which converges to some $u^{*} \in D$. We relabel the subsequence as the original sequence so that $T u_{n} \rightarrow u^{*}$ as $n \rightarrow \infty$. 
To conclude the proof of this theorem, we need to show that

$$
\lim _{n \rightarrow \infty}\left\|T u_{n}-u_{n}\right\|=0
$$

To that end, fixed $\theta=R$, and let $\varepsilon>0$ be give. By the integrability condition $\left(\mathrm{H}_{4}\right)$, there exists $0<\delta<1$ such that

$$
\int_{0}^{\delta} s(1-s)^{\alpha-2} f\left(s, q_{\theta}(s)\right) d s<\frac{\Gamma(\alpha)}{2} \varepsilon
$$

Further, by (3.11), there exists an $n_{0}$ such that, for $n \geq n_{0}$,

$$
v_{n}(t) \leq q_{\theta}(t) \text { on }[\delta, 1]
$$

so that

$$
v_{n}(t) \leq q_{\theta}(t) \leq u_{n}(t) \quad \text { on }[\delta, 1]
$$

Thus, for $s \in[\delta, 1]$ and $n \geq n_{0}$,

$$
f_{n}\left(s, u_{n}(s)\right)=f\left(s, \max \left\{u_{n}(s), v_{n}(s)\right\}\right)=f\left(s, u_{n}(s)\right),
$$

and for $t \in[0,1]$,

$$
\begin{aligned}
T u_{n}(t)-u_{n}(t) & =T u_{n}(t)-T_{n} u_{n}(t) \\
& =\int_{0}^{1} G(t, s)\left[f\left(s, u_{n}(s)\right)-f_{n}\left(s, u_{n}(s)\right)\right] d s .
\end{aligned}
$$

Thus, for $t \in[0,1]$,

$$
\begin{aligned}
\left|T u_{n}(t)-u_{n}(t)\right| & \leq \frac{1}{\Gamma(\alpha)}\left[\int_{0}^{\delta} s(1-s)^{\alpha-2} f\left(s, u_{n}(s)\right) d s+\int_{0}^{\delta} s(1-s)^{\alpha-2} f\left(s, \max \left\{u_{n}(s), v_{n}(s)\right\}\right) d s\right] \\
& \leq \frac{1}{\Gamma(\alpha)}\left[\int_{0}^{\delta} s(1-s)^{\alpha-2} f\left(s, u_{n}(s)\right) d s+\int_{0}^{\delta} s(1-s)^{\alpha-2} f\left(s, u_{n}(s)\right) d s\right] \\
& \leq \frac{2}{\Gamma(\alpha)} \int_{0}^{\delta} s(1-s)^{\alpha-2} f\left(s, q_{\theta}(s)\right) d s<\varepsilon .
\end{aligned}
$$

Since $t \in[0,1]$ was arbitrary, we conclude that $\left\|T u_{n}-u_{n}\right\| \leq \varepsilon$ for all $n \geq n_{0}$. Hence, $u^{*} \in\left[q_{R}, S\right]$ and for $t \in[0,1]$

$$
T u^{*}(t)=T\left(\lim _{n \rightarrow \infty} T u_{n}(t)\right)=T\left(\lim _{n \rightarrow \infty} u_{n}(t)\right)=\lim _{n \rightarrow \infty} T u_{n}=u^{*}(t) .
$$




\section{Acknowledgments}

The authors sincerely thank the reviewers for their valuable suggestions and useful comments that have led to the present improved version of the original paper. The Project Supported by the National Science Foundation of China (10971179) and Research Award Fund for Outstanding Young Scientists of Shandong Province (BS2010SF023).

\section{References}

[1] A. A. Kilbas, H. M. Srivastava, and J. J. Trujillo, Theory and Applications of Fractional Differential Equations, vol. 204 of North-Holland Mathematics Studies, Elsevier Science, Amsterdam, The Netherlands, 2006.

[2] K. S. Miller and B. Ross, An Introduction to the Fractional Calculus and Fractional Differential Equations, A Wiley-Interscience Publication, John Wiley \& Sons, New York, NY, USA, 1993.

[3] I. Podlubny, Fractional Differential Equations, vol. 198 of Mathematics in Science and Engineering, Academic Press, San Diego, Calif, USA, 1999.

[4] S. Zhang, "Existence of solution for a boundary value problem of fractional order," Acta Mathematica Scientia, vol. 26, no. 2, pp. 220-228, 2006.

[5] Z. Bai and H. Lü, "Positive solutions for boundary value problem of nonlinear fractional differential equation," Journal of Mathematical Analysis and Applications, vol. 311, no. 2, pp. 495-505, 2005.

[6] X. Xu, D. Jiang, and C. Yuan, "Multiple positive solutions for the boundary value problem of a nonlinear fractional differential equation," Nonlinear Analysis: Theory, Methods E Applications, vol. 71, no. 10, pp. 4676-4688, 2009.

[7] J. A. Gatica, V. Oliker, and P. Waltman, "Singular nonlinear boundary value problems for secondorder ordinary differential equations," Journal of Differential Equations, vol. 79, no. 1, pp. 62-78, 1989.

[8] J. Henderson and W. Yin, "Singular $(k, n-k)$ boundary value problems between conjugate and right focal," Journal of Computational and Applied Mathematics, vol. 88, no. 1, pp. 57-69, 1998.

[9] P. W. Eloe and J. Henderson, "Singular nonlinear $(k, n-k)$ conjugate boundary value problems," Journal of Differential Equations, vol. 133, no. 1, pp. 136-151, 1997.

[10] P. W. Eloe and J. Henderson, "Singular nonlinear multipoint conjugate boundary value problems," Communications in Applied Analysis, vol. 2, no. 4, pp. 497-511, 1998.

[11] J. J. DaCunha, J. M. Davis, and P. K. Singh, “Existence results for singular three point boundary value problems on time scales," Journal of Mathematical Analysis and Applications, vol. 295, no. 2, pp. 378-391, 2004.

[12] Y. Feng and S. Liu, "Solvability of a third-order two-point boundary value problem," Applied Mathematics Letters, vol. 18, no. 9, pp. 1034-1040, 2005.

[13] Q. Yao and Y. Feng, "The existence of solution for a third-order two-point boundary value problem," Applied Mathematics Letters, vol. 15, no. 2, pp. 227-232, 2002.

[14] K. Deimling, Nonlinear Functional Analysis, Springer, Berlin, Germany, 1985.

[15] D. J. Guo and V. Lakshmikantham, Nonlinear Problems in Abstract Cones, vol. 5 of Notes and Reports in Mathematics in Science and Engineering, Academic Press, Boston, Mass, USA, 1988. 


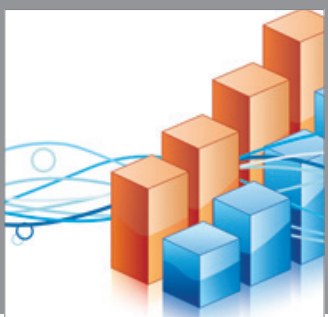

Advances in

Operations Research

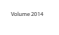

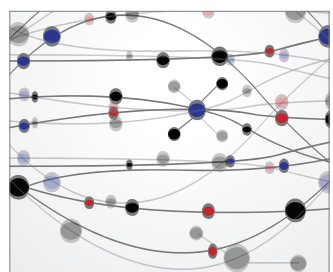

\section{The Scientific} World Journal
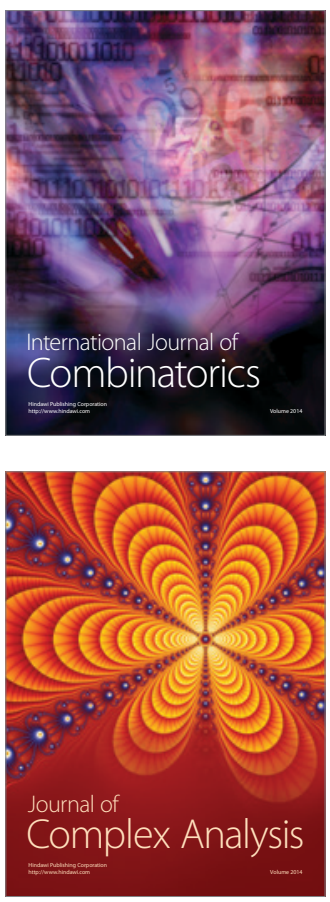

International Journal of

Mathematics and

Mathematical

Sciences
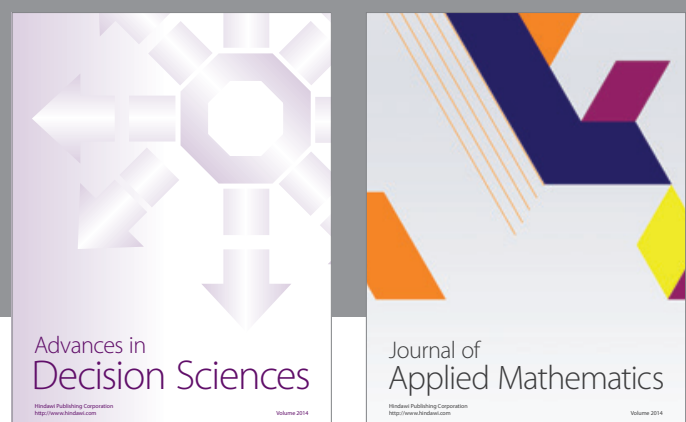

Journal of

Applied Mathematics
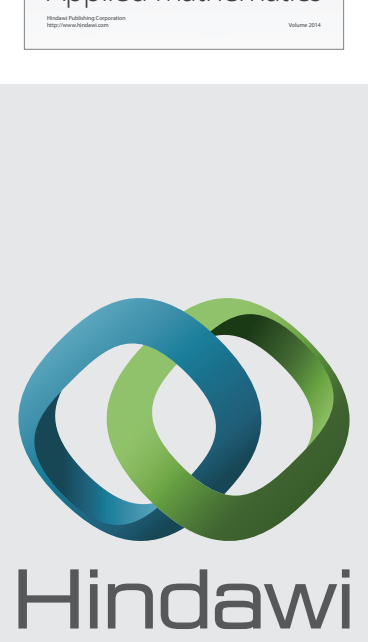

Submit your manuscripts at http://www.hindawi.com
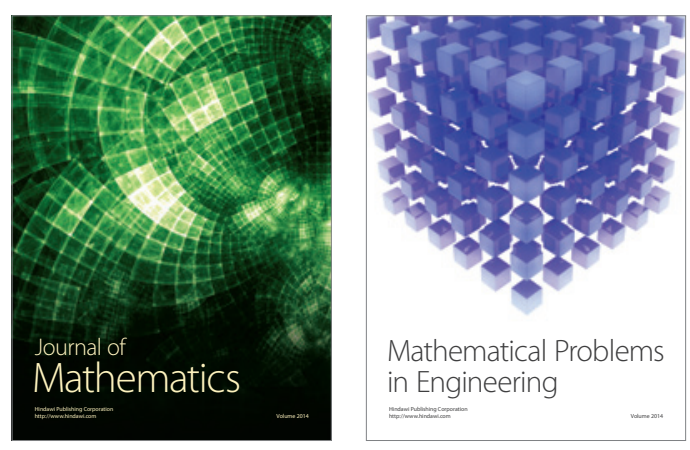

Mathematical Problems in Engineering
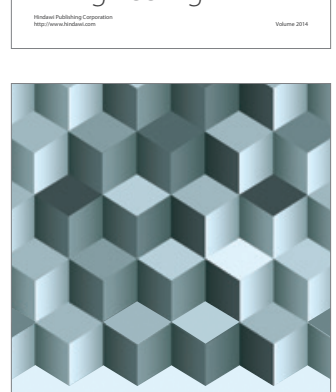

Journal of

Function Spaces
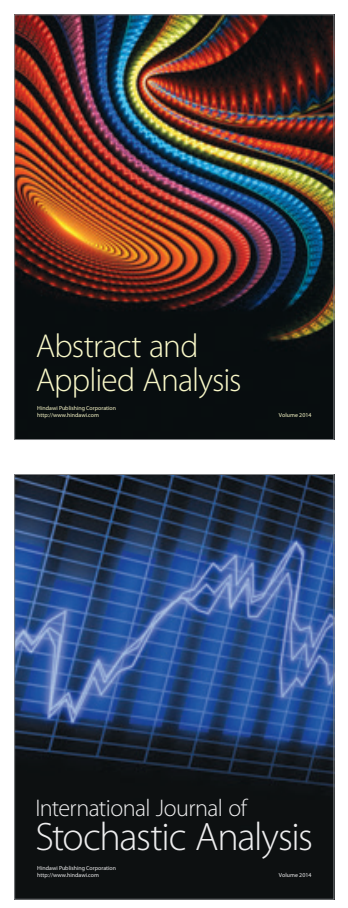

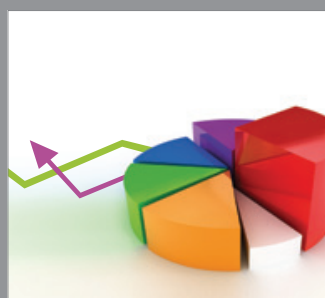

ournal of

Probability and Statistics

Promensencen
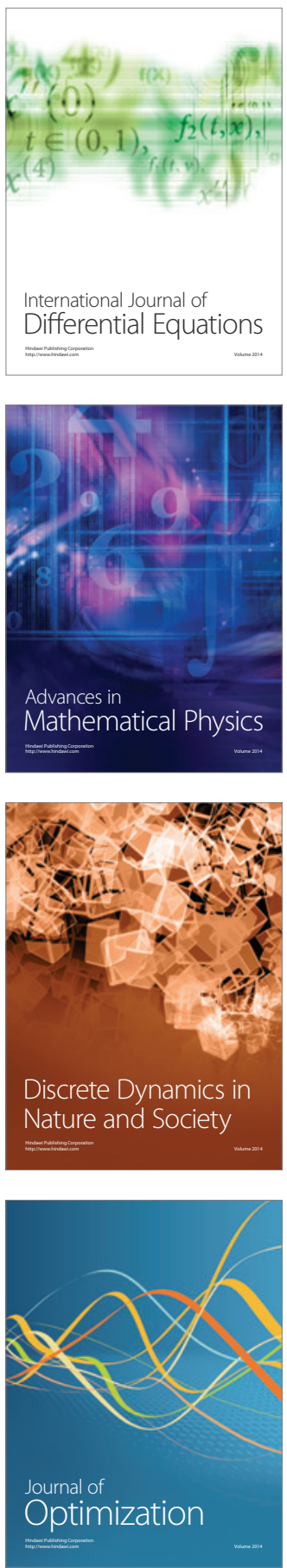